\title{
Local Glioma Cells Are Associated with Vascular Dysregulation
}

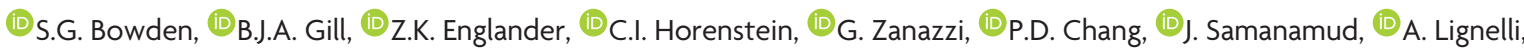 \\ (1).N. Bruce, (1)P. Canoll, and $\mathbb{1}^{\circ}$ J. Grinband
}

\begin{abstract}
BACKGROUND AND PURPOSE: Malignant glioma is a highly infiltrative malignancy that causes variable disruptions to the structure and function of the cerebrovasculature. While many of these structural disruptions have known correlative histopathologic alterations, the mechanisms underlying vascular dysfunction identified by resting-state blood oxygen level-dependent imaging are not yet known. The purpose of this study was to characterize the alterations that correlate with a blood oxygen level-dependent biomarker of vascular dysregulation.
\end{abstract}

MATERIALS AND METHODS: Thirty-two stereotactically localized biopsies were obtained from contrast-enhancing $(n=16)$ and nonenhancing $(n=16)$ regions during open surgical resection of malignant glioma in 17 patients. Preoperative resting-state blood oxygen level-dependent fMRI was used to evaluate the relationships between radiographic and histopathologic characteristics. Signal intensity for a blood oxygen level-dependent biomarker was compared with scores of tumor infiltration and microvascular proliferation as well as total cell and neuronal density.

RESULTS: Biopsies corresponded to a range of blood oxygen level-dependent signals, ranging from relatively normal $(z=-4.79)$ to markedly abnormal $(z=8.84)$. Total cell density was directly related to blood oxygen level-dependent signal abnormality $\left(P=.013, R^{2}=\right.$ $0.19)$, while the neuronal labeling index was inversely related to blood oxygen level-dependent signal abnormality $\left(P=.016, R^{2}=0.21\right)$. The blood oxygen level-dependent signal abnormality was also related to tumor infiltration $(P=.014)$ and microvascular proliferation $(P=$ .045).

CONCLUSIONS: The relationship between local, neoplastic characteristics and a blood oxygen level-dependent biomarker of vascular function suggests that local effects of glioma cell infiltration contribute to vascular dysregulation.

ABBREVIATIONS: $B$ OLD = blood oxygen level-dependent; $\mathrm{CE}=$ contrast-enhancing; $\mathrm{Gd}=$ gadolinium; $\mathrm{NE}=$ nonenhancing

M alignant glioma is a highly infiltrative malignancy that causes variable disruptions to the structure and function of the cerebrovasculature. ${ }^{1}$ Most prominent among these is a break-

\footnotetext{
Received February 8, 2017; accepted after revision November 9.

From the Department of Neurological Surgery (S.G.B.), Oregon Health \& Science University, Portland, Oregon; The Gabriele Bartoli Brain Tumor Research Laboratory (S.G.B., B.J.A.G., Z.K.E., J.S., J.N.B., P.C.), and Departments of Neurological Surgery (B.J.A.G., Z.K.E., J.N.B., P.C.), Pathology and Cell Biology (G.Z., P.C.), and Radiology (A.L., J.G.), Columbia University Medical Center, New York, New York; Department of Radiology (C.I.H.), North Shore University Hospital, Long Island, New York; and Department of Radiology (P.D.C.), University of California, San Francisco, California.

Preliminary results from this work were presented at: Eastern-Atlantic Medical Student Research Forum, March 3-5, 2016; Miami, Florida. Further results were previously presented at: Society of Neuro-Oncology Annual Meeting and Education Day, November 17-22, 2016; Scottsdale, Arizona.

This project was funded, in part, by the 2015 Research Scientist Award from the American Society of Neuroradiology (J.G.), the James S. McDonnell Foundation, and the Irving Institute for Clinical and Translational Research: Pilot Research Award (P.C.). Support from NVIDIA included 1 TITAN X Graphic Card (12 GB) to run a deep learning code to perform cell counting (P.D.C.). Dr Chang's salary is sup-
}

down of the blood-brain barrier, associated with the main tumor mass ${ }^{1-3}$ and clinically evident through the extravasation of gadolinium-conjugated contrast media. ${ }^{4}$ However, other vascular deficits are identifiable in the infiltrative margin of the tumor. Vasogenic edema, represented by T2-FLAIR hyperintensity, is the most frequently used abnormality for directing surgical and radiation treatments in nonenhancing (NE) areas. Recent application of functional imaging has identified additional physiologic consequences of malignant glioma infiltration, with decreased vascular reactivity noted on hypercapnia challenges ${ }^{5-7}$ and reduced cor-

\footnotetext{
ported by the National Institutes of Health (National Institute of Biomedical Imaging and Bioengineering) T32 Training Grant, T32EB001631.

Please address correspondence to Jack Grinband, PhD, Department of Radiology, Columbia University, 710 West 168th St, New York, NY 10032; e-mail: jackgrinband@gmail.com

- Indicates open access to non-subscribers at www.ajnr.org

三 Indicates article with supplemental on-line table.

http://dx.doi.org/10.3174/ajnr.A5526
} 
tical activation intensity and volume using task-based fMRI. ${ }^{8-11}$ Tumor vessel angiogenesis is a frequently cited and plausible contributor to such deficits within the contrast-enhancing (CE) tumor mass. ${ }^{5,7,9,10}$ However, areas of decreased vascular reactivity and reduced cortical activation have also been identified in lower grade gliomas, indicating that even sparsely infiltrated regions of brain parenchyma could have similar functional impairment. ${ }^{5}$ Nevertheless, the variable presence, distribution, and degree of these abnormalities leave their relationship to tumor infiltration uncertain.

A recent report by Chow et $\mathrm{al}^{12}$ identified a biomarker of glioma-related vascular dysregulation using resting-state blood oxygen level-dependent (BOLD) fMRI. The mean BOLD time-series extracted from normal, tumor-free brain of the contralesional hemisphere represents normal cardiac pulsations and respiration-related changes in arterial carbon dioxide $\left(\mathrm{CO}_{2}\right)^{13-15}$ and can be used as a temporal signature of normal vascular reactivity. ${ }^{16}$ Similarly, the mean BOLD time-series extracted from CE tumor represents abnormal tumor-related vascular function. By combining these temporal signatures, the spatial extent of gliomarelated vascular deficits can be illustrated by identifying voxels of NE brain parenchyma that behave more like the CE tumor and less like the normal contralesional brain tissue. This technique demonstrated that vascular dysregulation can extend beyond the CE tumor core into the NE infiltrative margin. Furthermore, the presence of nonoverlapping regions between the BOLD biomarker and T2-FLAIR hyperintensity, or BOLD-FLAIR mismatch, suggests discordance between deficits in neurovascular function and a breakdown of the blood-brain barrier. However, the absence of a histologic correlate in Chow et $\mathrm{al}^{12}$ leaves substantial uncertainty as to whether this vascular dysregulation is related directly to tumor infiltration or to the indirect effects of the tumor. For example, such dysfunction could be directly related to local tumor effects, such as a disruption in the structural, physical, or ultrastructural properties of the brain by invading glioma cells. ${ }^{11}$ Alternatively, distal, indirect causes, such as mass effect from the tumor or vasogenic edema; disruption of shared venous drainage; or damage to axons could also explain these BOLD abnormalities. ${ }^{8,12,17}$

The purpose of this study was to characterize the histopathologic alterations underlying the BOLD biomarker of vascular dysfunction in malignant gliomas. We compared BOLD dynamics from resting-state fMRI with neoplastic characteristics of a set of stereotactically localized biopsies. We hypothesized that the degree of disruption of vascular function measured by the BOLD biomarker is related to the severity of histopathologic abnormalities associated with tumor infiltration.

\section{MATERIALS AND METHODS \\ Patient Selection}

We performed a retrospective review of a database of MRI-localized biopsies obtained during open surgical resection of glioma at Columbia University Medical Center (CUMC). All patients included in the database previously provided consent as part of a CUMC review board-approved study protocol. Seventeen patients with pathology-proved, treatment-naïve glioblastoma $(n=$ $14)$ or grade III, $I D H$ wild-type astrocytoma $(n=3)$ and preop- erative BOLD fMRI were identified. The average age was 63.8 years (range, $48-82$ years) with 5 men and 12 women. Thirty-two tissue biopsies were obtained from the CE $(n=16)$ and the NE $(n=16)$ regions of tumor during open surgical resection. Automated cell counting was successfully performed on $31 \mathrm{H} \& \mathrm{E}$ stains and 28 NeuN stains (MAB377; MilliporeSigma, Darmstadt, Germany).

\section{Image Acquisition}

Before tumor resection, patients underwent standard-of-care imaging (including T1-weighted, gadolinium-enhanced [T1+Gd], T2-FLAIR, and DWI) as well as a resting-state BOLD fMRI scans on 1 of 4 hospital scanners (Intera 1.5T, Achieva 3T, Philips Healthcare, Best, the Netherlands; TwinSpeed HDx 1.5T or Excite HDx 3T, GE Healthcare, Milwaukee, Wisconsin). The ranges of scanning parameters for $\mathrm{T} 1$ sequences were the following: $\mathrm{TR}=$ $10-2000 \mathrm{~ms}$, flip angle $=13^{\circ}-90^{\circ}, \mathrm{TE}=2-20 \mathrm{~ms}$. For FLAIR sequences, the ranges were the following: $\mathrm{TR}=8000-11,000 \mathrm{~ms}$, flip angle $=90^{\circ}, \mathrm{TE}=104-337 \mathrm{~ms}$, TI $=2200-2800 \mathrm{~ms}$. BOLD parameters were the following: $\mathrm{TR}=1000-2400 \mathrm{~ms}$, flip angle $=$ $72^{\circ}-90^{\circ}, \mathrm{TE}=20-34 \mathrm{~ms}$, number of volumes $=150-200$. The scanning parameters for each patient are listed in the On-line Table. Contrast-enhanced images were acquired with IV gadobenate dimeglumine (MultiHance; Bracco Diagnostics, Princeton, New Jersey) with a weight-based dose of $0.2 \mathrm{~mL} / \mathrm{kg}$. The time between the IV injection and contrast-enhanced imaging was 5 minutes. BOLD images were obtained before contrast-enhanced imaging.

\section{Biopsy Acquisition}

All tissue sampling was performed during the normal surgical plan, provided it posed no additional risk to the patient. Samples were taken from the CE tumor core in all cases and NE regions when within the planned surgical trajectory. Biopsies were obtained before surgical debulking to minimize the effects of brain shift and deformation. Frameless stereotactic guidance was provided by a volumetric T1 + Gd scan uploaded to a neuronavigation interface (Curve; Brainlab, Feldkirchen, Germany). Biopsy location was recorded by screen captures of the neuronavigation interface, allowing the downstream determination of the Cartesian coordinates of each biopsy. We have recently shown that this method results in spatial accuracy to within $\sim 2 \mathrm{~mm} .{ }^{18}$ Biopsies that were taken from radiologically defined areas of necrosis or those that were predominantly histologically necrotic ( $>75 \%$ of the tissue sample) were excluded from the analysis.

\section{Histopathologic Analysis}

Samples were formalin-fixed and paraffin-embedded for histopathologic analysis. Five-micrometer sections were stained with H\&E. Seven-micrometer sections were immunostained with the NeuN antibody. Qualitative histologic characteristics of neoplasms were evaluated by 2 blinded neuropathologists (G.Z., P.C.). H\&E-stained sections were reviewed for the degree of tumor infiltration and scored as no tumor present (score $=0$ ), infiltrating tumor (score $=1$ ), or highly cellular tumor (score $=$ 2). The tumor score was a composite of several features, including cytologic atypia and cell density. H\&E slides were also reviewed 
for the degree of microvascular proliferation, scored as delicate microvasculature (normal vascular architecture; score $=0$ ), simple hyperplastic structures ( $<3$ circumferential layers with patent lumen; score $=1)$, or complex microvascular hyperplasia $(\geq 3$ endothelial layers or glomeruloid-type vessels; score $=2$ ).

Total cell density was calculated using an automated cellcounting algorithm. ${ }^{18}$ Briefly, the H\&E-stained sections were digitalized using a Leica scn400 system (Leica Biosystems, Buffalo Grove, Illinois), with hematoxylin-stained nuclei counted in iteratively processed high-power fields. Total cell density was computed as the median number of hematoxylin-stained cells across all high-power fields that contained at least 1 nucleus. Each immunoperoxidase-stained slide was digitized at low-power $\times 10$ magnification, and a semiautomated algorithm was used to determine the overall NeuN index, to calculate the labeling index for NeuN. For each slide, user-defined RGB (3-channel) and size thresholds were determined by visual inspection of 9 randomly generated low-power $\times 10$ fields. On the basis of these statistics, the algorithm subsequently identified all immunoperoxidasestained cells in each low-power field and computed the neuronal fraction by dividing the surface area of stained cells by the total surface area of all tissue in the low-power field. The NeuN index was defined as the $90 \%$ quantile of fractions across all low-power fields that contained at least 1 stained cell.

\section{Screenshot Registration and Coordinate Determination}

Screenshots of the neuronavigation interface showing the biopsy location were superimposed onto a coregistered T1+Gd image. Coregistration of the screenshots to the $\mathrm{T} 1+\mathrm{Gd}$ image in $3 \mathrm{D}$ space was performed using custom software written in Matlab (MathWorks, Natick, Massachusetts). The registration pipeline first correlated the pixel intensities of the screenshot to the voxel intensities of each volumetric T1 slice, and the slice with peak correlation was identified as the biopsy slice. A correlation between the screenshot and the biopsy slice was then taken at different $x$ and $y$ offsets, and the peak correlation was used to identify the correct $x$ and $y$ offsets. Finally, each biopsy location on the screenshot was labeled with a cross-hair. To identify this location, we correlated a digital cross-hair to the screenshot at different $\mathrm{x}$ and $y$ offsets and used the peak correlation to identify the $x$ and $y$ coordinates of the biopsy site. All coregistered screenshots were confirmed by visual inspection.

\section{Tumor Masks}

All structural images were coregistered to the T1 + Gd image with the highest spatial resolution using rigid-body registration (FMRIB Linear Image Registration Tool, FLIRT; https://fsl. fmrib.ox.ac.uk/fsl/fslwiki/FLIRT ${ }^{19} ; 6 d f$ ), and all masks were created in the high-resolution reference image space. 3D masks of the CE tumor were hand drawn on high-resolution $\mathrm{T} 1+\mathrm{Gd}$ images by a board-certified neuroradiologist (C.I.H.). The boundaries of the masks were drawn to be as specific for CE intra-axial tumor as possible. To this end, we excluded the following from the tumor mask: ambiguous voxels at the border of CE tumor and NE brain tissue; ambiguous voxels at the border of CE tumor and necrotic core; areas of linear enhancement determined to represent vessels; and extra-axial spread of tumor along the dura or ventricular surface. The control masks were generated by coregistering the T1+Gd image to Montreal Neurological Institute 152 space and transforming a contralesional hemisphere mask, created in Montreal Neurological Institute 152 space, to the patient's reference space (ie, the high-resolution $\mathrm{T} 1+\mathrm{Gd}$ image) using nearestneighbor interpolation. The contralesional control masks were then visually inspected by a board-certified neuroradiologist (C.I.H.) to ensure that no CE tumor or abnormal FLAIR hyperintense tissue was included in the mask. To generate the functional masks, we registered BOLD scans to the high-resolution reference image (FLIRT, $6 d f$ ) and applied the inverse transform to the high-resolution masks to put them into functional space. Because the BOLD data were collected at a lower spatial resolution than the structural data, the transformation of the masks resulted in partial voluming. To minimize any influence of partial voluming from nonenhancing portions of the surrounding tissue, the CE masks were thresholded so that the fraction of the CE tumor was at least 0.95 . Similarly, the control masks were thresholded so that the fraction of NE tissue was at least 0.95 . All original and transformed masks were visually inspected by a board-certified radiologist (C.I.H.) to confirm accuracy.

\section{Image Preprocessing of BOLD Data}

All functional data were processed with the FMRIB Software Library (FSL, Version 5.0.6; http://www.fmrib.ox.ac.uk/fsl) ${ }^{19}$ and Matlab (2012b) software. Each functional image was motion-corrected, slice-timing corrected, spatially smoothed (Gaussian filter, full width at half maximum $=5 \mathrm{~mm})$, high-pass filtered $(100$ seconds), and skull-stripped. We performed independent components analysis (MELODIC; https://fsl.fmrib.ox.ac.uk/fsl/ fslwiki/MELODIC ${ }^{20}$ ), which resulted in $\sim 40-90$ components per scan. The components were visually inspected, and any components related to scanner noise or head movement artifacts were removed. To further minimize the effect of motion on BOLD signal intensity, the motion parameters were regressed out of the data before additional regression was performed with the tumor and control ROI time-series. The 18 motion regressors used in the regression model consisted of 3 translations, 3 rotations, the first derivative of each, and the corresponding quadratic terms. The residual from this model was then used for all subsequent regression analyses.

\section{BOLD Dynamics}

To create the BOLD masks, we followed the procedure described in Chow et al. ${ }^{12}$ We first made the simplifying assumption that there are 2 functional tissue classes within the brain, tumor and healthy tissue, which are represented by the CE portion of the tumor and the contralesional hemisphere, respectively. Mean time-series were extracted from the masks defined by the CE tumor and from the contralesional control hemisphere, by averaging the BOLD time-series of all voxels within each mask. Multiple linear regression was performed for all brain voxels using the 2 -regressor model, the CE tumor mean time-series and the contralesional control mean time-series and their first derivatives, to allow variation in timing. The output of this analysis and the metrics used to make our conclusions consisted of parameter estimates ( $\beta$ weights) and $z$ statistic values ( $\beta$ weights normalized by 


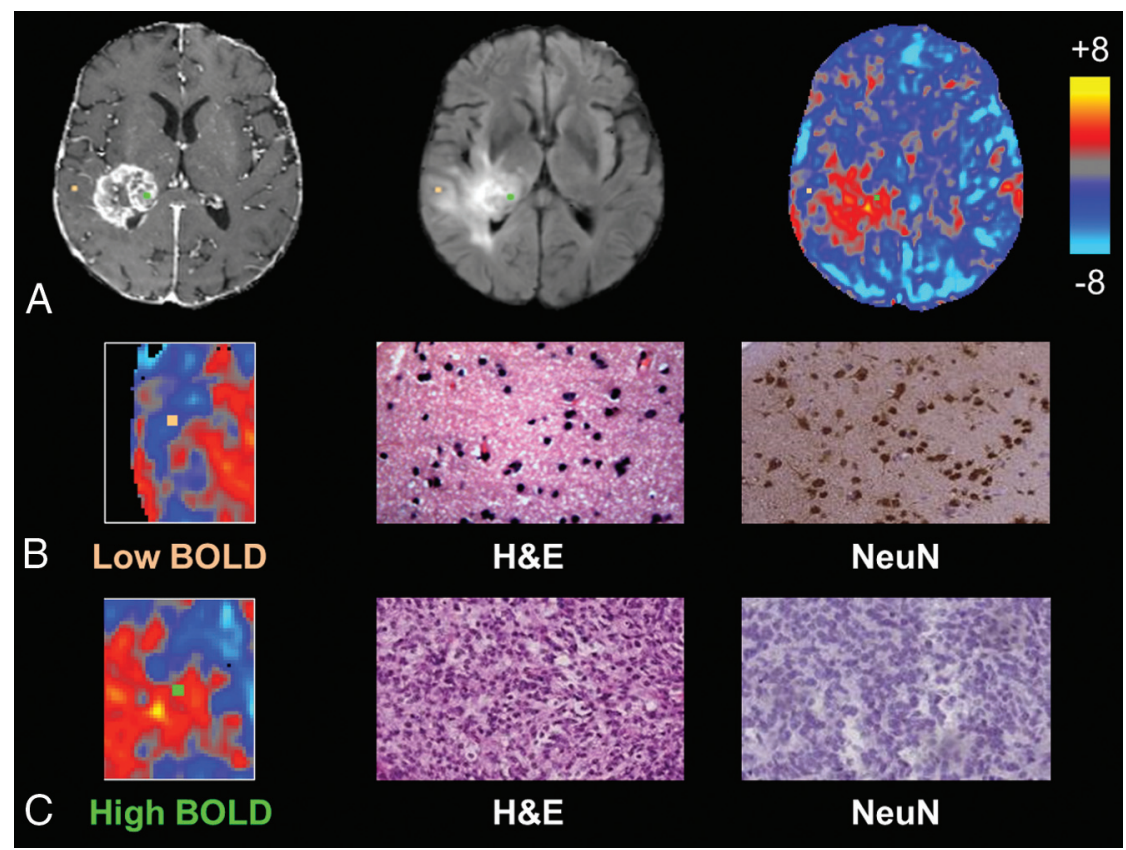

FIG 1. Individual biopsies from areas of intact and disrupted vascular function exhibit distinct histopathologic characteristics. A, Biopsy locations overlaid onto T1 postcontrast, FLAIR, and BOLD images with correlative histopathology in $B$ and $C$. The sample from relatively normal BOLD signal, representing normal vascular function (orange, $B$ ), shows normal cellularity and high NeuN staining, suggestive of noninfiltrated gray matter, whereas the sample from a region of BOLD signal abnormality (green, $C$ ) shows tumor tissue and no neuronal staining.

the residual variance) for each voxel. The difference between the 2 $z$ statistic images (ie, $Z_{\mathrm{CE}}-Z_{\text {Control }}$ ) was used as the BOLD biomarker representing vascular function, as described in Chow et al. ${ }^{12}$ Specifically, strongly negative values (shown in blue on $z$ statistic maps, Fig 1) represent areas of normal vascular function-that is, the voxel time-series is more like the control ROI; strongly positive values (shown in red-yellow on $z$ statistic maps, Fig 1) represent areas of vascular dysregulation - that is, the voxel time-series is more like the CE tumor.

\section{Analysis of Histopathologic and Radiologic Correlation}

All statistical tests were performed using the Statistics Toolbox in Matlab (2012b). Linear regression was used to evaluate the relationships between BOLD signal intensity and quantitative histopathologic features (total cell density and NeuN labeling index). The parameter estimate for the CE regressor decreases as a function of distance from the CE mask due to tumor-related vascular dysregulation and nonspecific effects of distance (ie, voxels in close proximity have more similar intensities than voxels that are far apart). To control for nonspecific effects of distance, we included a distance regressor, the measured distance from the biopsy location to the nearest $\mathrm{CE}$ voxel, in the regression model of cellularity versus BOLD and NeuN versus BOLD. To assess the relationship between qualitative histologic features (degree of tumor infiltration and microvascular proliferation) and the BOLD signal, we performed a 1-way analysis of variance.

\section{RESULTS}

Biopsies demonstrated a range of BOLD signal intensities based on their projection onto $z$ statistic maps, ranging from relatively normal $(z=-4.79)$ to markedly abnormal $(z=8.84)$. Differences in the degree of signal abnormality appeared to be related to certain histopathologic characteristics within individual tissue samples. Representative biopsies from a single patient are shown in Fig 1, which illustrates tissue samples taken from areas of differing BOLD signals (Fig $1 A$ ). Tissue corresponding to a negative BOLD value $(z=-1.90$; Fig $1 B)$ demonstrated normal cell density, high NeuN staining, noninfiltrated gray matter, and normal, delicate microvasculature. In contrast, a sample corresponding to a highly positive BOLD signal $(z=4.44$; Fig $1 C$ ) contained hypercellular tissue, little neuronal staining, highly cellular tumor, and complex microvascular hyperplasia.

Among all biopsies, linear regression analysis demonstrated a significant inverse relationship between total cell density and distance from the nearest CE voxel $\left(P=.003, R^{2}=0.27\right.$; Fig $\left.2 A\right)$ and a positive relationship between neuronal density and distance $\left(P=.032, R^{2}=0.17\right.$; Fig $\left.2 B\right)$. The BOLD signal also showed an inverse relationship with distance $\left(P=.004, R^{2}=0.25\right.$; Fig $\left.2 C\right)$. Linear regression between BOLD signal intensity and total cell density, with distance to the nearest $\mathrm{CE}$ voxel as a confounding variable, showed a positive relationship $\left(P=2 \times 10^{-5}, R^{2}=0.48\right.$; Fig $3 A$ ). A similar analysis showed that the NeuN labeling index was negatively related to BOLD signal intensity $\left(P=.001, R^{2}=\right.$ 0.35 ; Fig $3 B$ ). An alternative explanation for this negative relationship could be the greater prevalence of gliomas in white matter, which normally has low neuronal density, rather than the neurotoxic effects of the tumor. To test this hypothesis, we compared the BOLD values in gray (mean, $-2.9 \pm 0.76$ ) and white (mean, $-2.4 \pm 0.83$ ) matter on the contralesional control hemisphere. Gray matter voxels were significantly more negative than white matter voxels ( $t$ test, $P=.001, t=4.00$ ); however, the difference in BOLD values due to tissue type (gray-white $=-0.54 \pm$ 0.56) cannot explain the very large range of BOLD values 

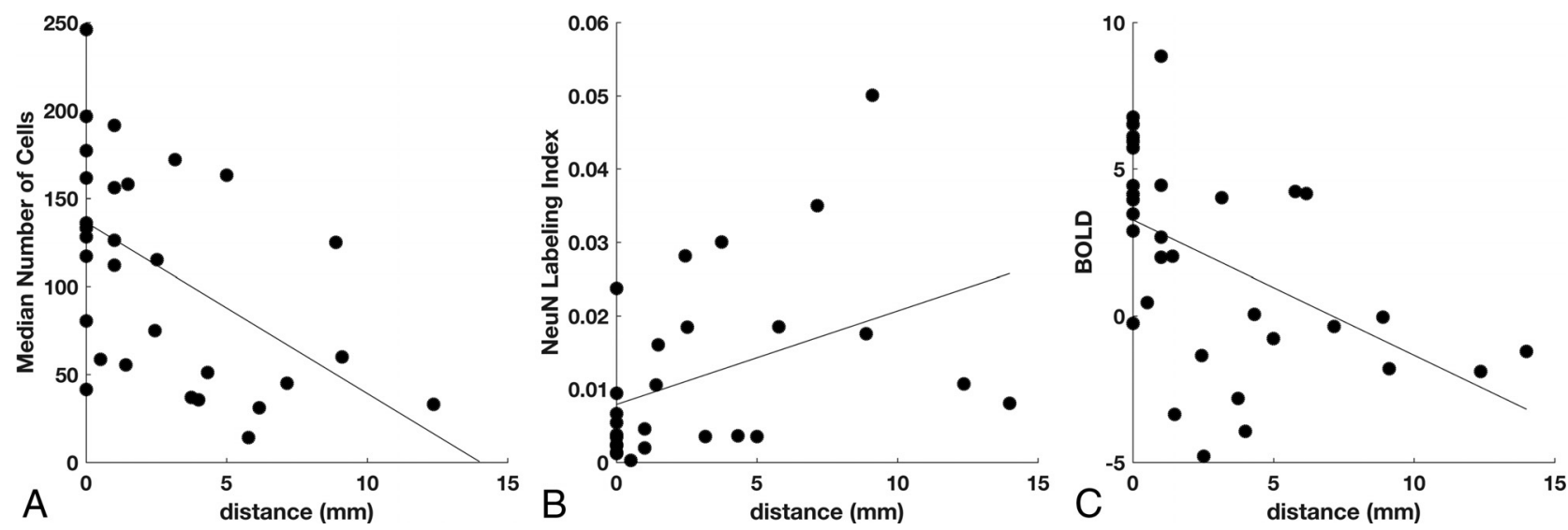

FIG 2. Effect of distance from contrast enhancement on cellularity, neuronal density, and BOLD signal abnormality. $A$, Cell density decreases with the distance to the nearest contrast-enhancing voxel $(n=31, P=.003)$. $B$, Neuronal density increases with the distance to contrast enhancement $(n=27, P=.032)$. $C$, Intensity of the BOLD biomarker decreases with the distance to contrast enhancement $(n=32, P=.004)$.
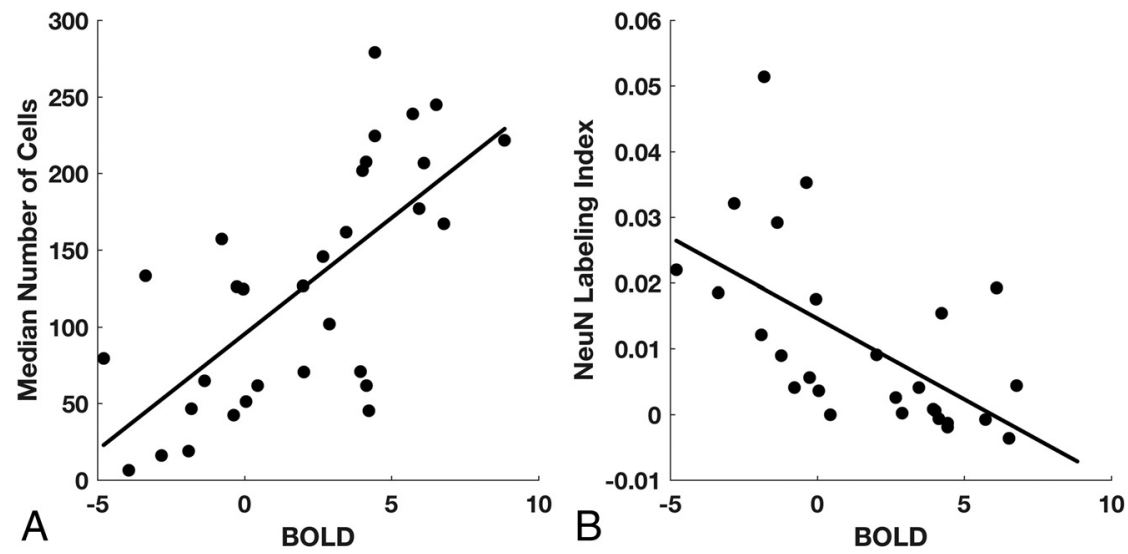

FIG 3. BOLD signal abnormality is related to total cell and neuronal density. A, After we controlled for the effect of distance on the BOLD signal, median cell density is positively related to the BOLD z statistic $\left(n=31, P=2 \times 10^{-5}\right)$. B. The NeuN labeling index is inversely related to the BOLD $z$ statistic $(n=27, P=.001)$.

across the biopsy samples (maximum-minimum $=8.85$ to $-4.79=13.64)$.

The distributions of BOLD signal abnormality related to tumor infiltration and microvascular proliferation are summarized in Fig 4. Across all biopsies, both the tumor infiltration score (ANOVA: $F[2,29]=4.95, P=.014)($ Fig $4 A)$ and the microvascular proliferation score (ANOVA: $F[2,29)]=3.46, P=.045$ ) (Fig $4 B$ ) were significantly related to the BOLD signal abnormality. A post hoc Student $t$ test showed a significant difference in BOLD signal between the group of samples with no detectable tumor $($ score $=0)$ and those with highly cellular tumor (score $=$ $2 ; P=.003$ ) or the combination of infiltrating and highly cellular tumor (score 1 or $2 ; P=.029$ ). Similarly, a $t$ test showed a significant difference in BOLD signal between the group of samples with no identifiable microvascular proliferation $($ score $=0)$ and those with marked vascular hyperplasia (score $=2 ; P=.038$ ) or the combination of simple and complex vascular hyperplasia (score 1 or $2 ; P=.027$ ).

\section{DISCUSSION}

The diffuse infiltration and histologic heterogeneity of malignant gliomas pose a distinct challenge to the identification of the toxic effects of these tumors on the structure and function of the cere- brovasculature. The integrity of the vasculature in and around the main mass of the tumor is typically assessed with standard-of-care imaging that uses T1-weighted contrast-enhanced and FLAIR sequences. ${ }^{1-4}$ Regions of abnormal contrast enhancement associated with the main tumor mass typically correspond to elevated $\mathrm{CBV}, \mathrm{CBF}$, and microvascular permeability on perfusionweighted imaging. ${ }^{21-27}$ Most interesting, there are few vascular abnormalities in the NE, FLAIR-hyperintense peritumoral regions on perfusion imaging, ${ }^{21-27}$ despite the recognized infiltration of tumor cells into these areas. ${ }^{28-31}$ Additional evidence suggests that edema surrounding the tumor originates directly from the CE tissue, passively diffuses into the NE regions, and may therefore not be associated with blood-brain barrier breakdown in the NE tissue. ${ }^{32,33}$

Several intriguing studies have, however, identified decreased vascular reactivity outside CE regions of glioma. ${ }^{5,7,34}$ In turn, it has been postulated that infiltrating glioma cells disrupt neurovascular coupling, ${ }^{35,36}$ impairing both normal hypercapnia-induced elevations in CBF and CBV and the vascular reactivity of cortical tissue, particularly within gray matter. However, without any histopathologic correlation to these radiologic findings, it has been unclear whether infiltrating glioma cells contribute directly 

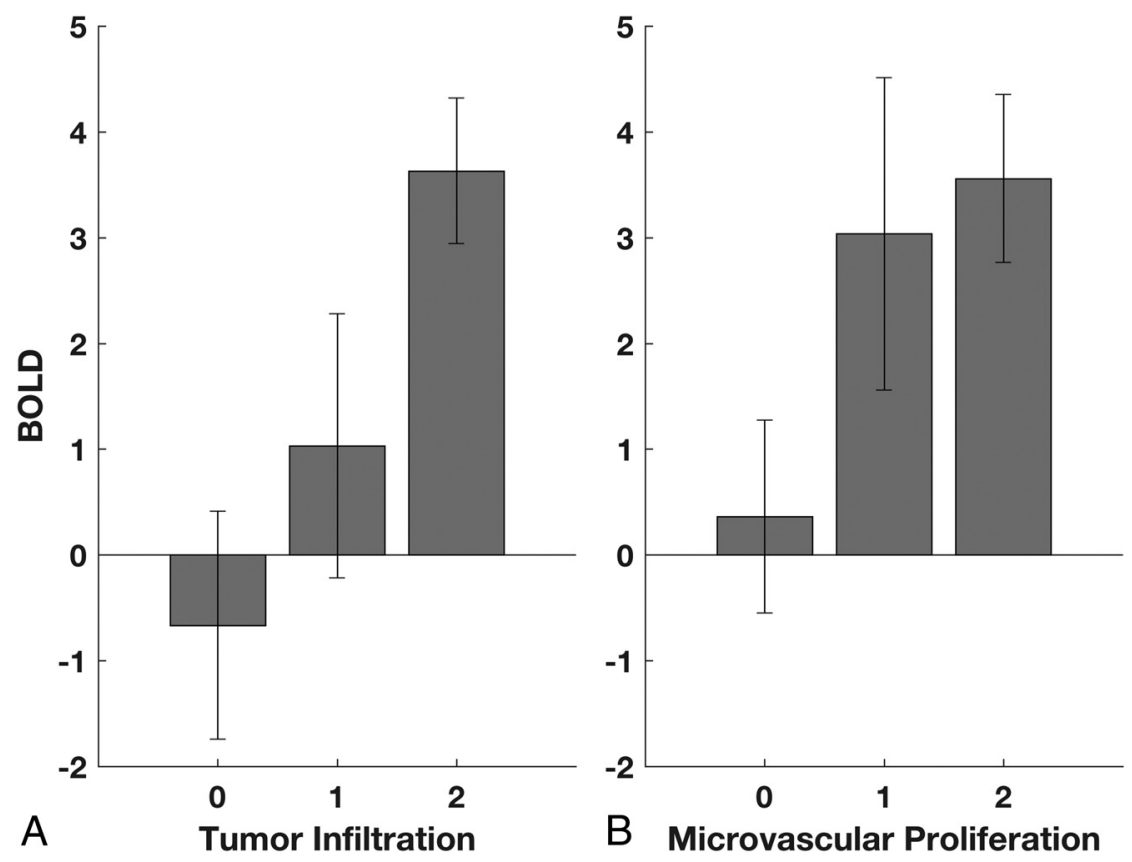

FIG 4. BOLD signal abnormality is related to tumor infiltration and microvascular proliferation. $A$, ANOVA demonstrates a significant relationship between the BOLD $z$ statistic and tumor infiltration $(P=.014)$. Scores of 0,1 , and 2 represent no detectable tumor, infiltrating tumor, and highly cellular tumor, respectively. B, ANOVA demonstrates a significant relationship between the BOLD $z$ statistic and microvascular proliferation $(P=.045)$. Scores of 0,1 , and 2 represent normal vascular architecture, simple hyperplastic structures, and complex microvascular hyperplasia, respectively.

to these abnormalities. The results of the present study, by relating histopathologic and radiologic data, demonstrate that the BOLD biomarker of vascular dysfunction is directly associated with tumor infiltration. We found markedly abnormal tissue in areas of BOLD signal abnormality and relatively unaffected brain tissue in areas of relatively normal BOLD signal. Moreover, the BOLD biomarker is directly related to total cell density and inversely related to neuronal density and is correlated to scores of tumor infiltration and microvascular proliferation.

The spatial distribution of the BOLD biomarker is also consistent with a decreasing cell density gradient with distance from the tumor core. ${ }^{28}$ The biomarker decreases as a function of distance from the CE tumor core, replicating our previous findings. ${ }^{12} \mathrm{We}$ found that the effect of distance is similar among BOLD, cellularity, and neuronal density, suggesting that the detectable infiltrating margins of the tumor span approximately $1-2 \mathrm{~cm}$ beyond the contrast enhancement, comparable with the commonly used 2- to $3-\mathrm{cm}$ margins for radiation treatment. ${ }^{37}$ More important, the relationship between the BOLD biomarker and the cell counts (ie, cellularity and neuronal density) remained statistically significant, even after including distance from the CE core as a covariate, indicating that the BOLD data can explain additional variance of tumor heterogeneity unrelated to a simple density gradient.

The mechanisms by which glioma influences vascular function are unknown. Several potential mechanisms have been proposed as broad or long-distance effects of glioma on vascular function. For example, mass effect and consequent venous compression could alter rates of blood flow over large regions of surrounding brain tissue ${ }^{8,17}$; shared venous drainage could allow signal from blood draining from the tumor core to predominate even in the peritumoral area ${ }^{12}$; the effects of aberrant metabolite and neurotransmitter production found in gliomas may extend to normal cortical vessels, inducing vascular dysregulation at indeterminate distances from invading tumor cells; and the tumor may affect fibers of passage in its vicinity, inducing necrosis or structural changes with detrimental downstream effects, consistent with findings of multidomain cognitive dysfunction in some patients with glioblastoma that are not easily attributable to local injury from the tumor ${ }^{38}$ and the disruption of global resting-state networks. ${ }^{39-42}$

In contrast, local tumor effects may also contribute to the vascular dysfunction seen with the BOLD biomarker. For example, invading glioma cells could perturb the structure of the neurovascular unit by compromising direct contact between vascular endothelial cells and astrocytes. ${ }^{1,3,43}$ Alternatively, astrocytic mediation of the vascular response may be modulated directly by the neurotransmitters of the glioma cells ${ }^{44}$ or by affecting the action of normal signaling molecules.

A BOLD fMRI biomarker of vascular dysfunction has several potential clinical applications that could warrant further investigation. BOLD, with its sensitivity to local effects of infiltrating glioma, could be another useful adjunct in guiding surgery beyond the CE boundary, especially considering the growing body of evidence favoring aggressive resection. ${ }^{45,46}$ For the same reason, it may be useful in targeting adjuvant radiation therapy toward affected areas unamenable to surgical resection. In the same vein, investigating the BOLD dynamics in areas of residual disease may offer insight into the mechanisms of disease recurrence and progression: It is possible that disease recurrence is more attributable to compromised vascular function than residual tumor burden alone. Thus, application of the BOLD biomarker in posttreatment surveillance could offer new insight into patterns of 
glioblastoma spread, assess response to new therapies, and segment areas of tumor recurrence.

There are, however, several limitations to our conclusions. There is no way to ensure perfect fidelity between the physical biopsy location and the biopsy site as represented by frameless stereotaxy during an open tumor resection. As per the study protocol, attempts were made to mitigate the effects of intraoperative brain shift within the limits of the surgical plan by minimizing intraoperative use of mannitol, CSF diversion, or hyperventilation before biopsy. Most important, all biopsy sampling was performed before tumor debulking. Nevertheless, it is possible that the BOLD value extracted from the presumed biopsy site could be displaced by $\geq 1$ voxel from the true biopsy location. However, we recently showed that our sampling method has registration accuracy to within $\sim 2 \mathrm{~mm},{ }^{18}$ suggesting that this type of error would have minimal effect on our results.

Additionally, we did not have rigid control of scanning parameters due to the retrospective nature of this study; consequently, contrast-to-noise and signal-to-noise could vary significantly across individuals. However, all structural data were reviewed by a neuroradiologist and assessed to be clearly interpretable for creating tissue masks. Similarly, variability in scanning parameters certainly affected the signal-to-noise properties of the BOLD data. Despite this variability, we showed a significant relationship between BOLD data and histologic features, suggesting that future prospective studies that can hold scanning parameters constant could decrease the variance of this relationship. Finally, masks were constructed by a single neuroradiologist, resulting in some subjectivity in mask boundaries. However, our previous work ${ }^{12}$ has demonstrated that the vascular dysregulation maps are not sensitive to small changes in mask variability (eg, using gray matter versus white matter as the contralesional control mask resulted in 2 BOLD maps that had a correlation of 0.96).

\section{CONCLUSIONS}

Vascular dysregulation, as represented by a resting-state BOLD biomarker, is seen both within and well beyond the margins of contrast enhancement in malignant gliomas. Many factors likely influence this dysfunction in NE regions. However, the statistically significant correlation between tumor infiltration and the degree of BOLD abnormality suggests that infiltrating glioma cells contribute to vascular dysfunction in both $\mathrm{CE}$ and NE regions.

Disclosures: Peter D. Chang—RELATED: Provision of Writing Assistance, Medicines, Equipment, or Administrative Support: NVIDIA Corporation, Comments: donation of graphics-processing unit computing hardware for analysis. Peter Canoll-RELATED: Grant: James S. McDonnell Foundation, Comments: This grant supported the collection and analysis of the localized biopsies*. Jack Grinband-RELATED: Grant: American Society of Neuroradiology*. *Money paid to the institution.

\section{REFERENCES}

1. Watkins S, Robel S, Kimbrough IF, et al. Disruption of astrocytevascular coupling and the blood-brain barrier by invading glioma cells. Nat Commun 2014;5:4196 CrossRef Medline

2. Persidsky Y, Ramirez SH, Haorah J, et al. Blood-brain barrier: structural components and function under physiologic and pathologic conditions. J Neuroimmune Pharmacol 2006;1:223-36 CrossRef Medline

3. Cuddapah VA, Robel S, Watkins S, et al. A neurocentric perspective on glioma invasion. Nat Rev Neurosci 2014;15:455-65 CrossRef Medline

4. Wen PY, Macdonald DR, Reardon DA, et al. Updated response assessment criteria for high-grade gliomas: Response Assessment in Neuro-Oncology working group. J Clin Oncol 2010;28:1963-72 CrossRef Medline

5. Pillai JJ, Zacà D. Comparison of BOLD cerebrovascular reactivity mapping and DSC MR perfusion imaging for prediction of neurovascular uncoupling potential in brain tumors. Technol Cancer Res Treat 2012;11:361-74 CrossRef Medline

6. Hsu YY, Chang CN, Jung SM, et al. Blood oxygenation level-dependent MRI of cerebral gliomas during breath holding. J Magn Reson Imaging 2004;19:160-67 CrossRef Medline

7. Zacà D, Jovicich J, Nadar SR, et al. Cerebrovascular reactivity mapping in patients with low grade gliomas undergoing presurgical sensorimotor mapping with BOLD fMRI. J Magn Reson Imaging 2014;40:383-90 CrossRef Medline

8. Holodny AI, Schulder M, Liu WC, et al. The effect of brain tumors on BOLD functional MR imaging activation in the adjacent motor cortex: implications for image-guided neurosurgery. AJNR Am J Neuroradiol 2000;21:1415-22 Medline

9. Hou BL, Bradbury M, Peck KK, et al. Effect of brain tumor neovasculature defined by rCBV on BOLD fMRI activation volume in the primary motor cortex. Neuroimage 2006;32:489-97 CrossRef Medline

10. Jiang Z, Krainik A, David O, et al. Impaired fMRI activation in patients with primary brain tumors. Neuroimage 2010;52:538-48 CrossRef Medline

11. Schreiber A, Hubbe U, Ziyeh S, et al. The influence of gliomas and nonglial space-occupying lesions on blood-oxygen-leveldependent contrast enhancement. AJNR Am J Neuroradiol 2000;21: 1055-63 Medline

12. Chow DS, Horenstein CI, Canoll P, et al. Glioblastoma induces vascular dysregulation in nonenhancing peritumoral regions in humans. AJR Am J Roentgenol 2016;206:1073-81 CrossRef Medline

13. Shmueli K, van Gelderen P, de Zwart JA, et al. Low-frequency fluctuations in the cardiac rate as a source of variance in the restingstate fMRI BOLD signal. Neuroimage 2007;38:306-20 CrossRef Medline

14. Murphy K, Birn RM, Handwerker DA, et al. The impact of global signal regression on resting state correlations: are anti-correlated networks introduced? Neuroimage 2009;44:893-905 CrossRef Medline

15. Birn RM, Murphy K, Bandettini PA. The effect of respiration variations on independent component analysis results of resting state functional connectivity. Hum Brain Mapp 2008;29:740-50 CrossRef Medline

16. Liu $\mathrm{P}, \mathrm{Li} Y$, Pinho $M$, et al. Cerebrovascular reactivity mapping without gas challenges. Neuroimage 2017;146:320-26 CrossRef Medline

17. Holodny AI, Schulder M, Liu WC, et al. Decreased BOLD functional MR activation of the motor and sensory cortices adjacent to a glioblastoma multiforme: implications for image-guided neurosurgery. AJNR Am J Neuroradiol 1999;20:609-12 Medline

18. Chang PD, Malone HR, Bowden S, et al. A multiparametric model for mapping cellularity in glioblastoma using radiographically localized biopsies. AJNR Am J Neuroradiol 2017;38:890-98 CrossRef Medline

19. Jenkinson M, Beckmann CF, Behrens TE, et al. FSL. Neuroimage 2012;62:782-90 CrossRef Medline

20. Beckmann CF, Smith SM. Probabilistic independent component analysis for functional magnetic resonance imaging. IEEE Trans Med Imaging 2004;23:137-52 CrossRef Medline

21. Calli C, Kitis O, Yunten N, et al. Perfusion and diffusion MR imaging in enhancing malignant cerebral tumors. Eur J Radiol 2006;58:394403 CrossRef Medline

22. Hakyemez B, Erdogan C, Bolca N, et al. Evaluation of different cerebral mass lesions by perfusion-weighted MR imaging. JMagn Reson Imaging 2006;24:817-24 CrossRef Medline

AJNR Am J Neuroradiol 39:507-14 Mar 2018 www.ajnr.org 
23. Tyler JL, Diksic M, Villemure JG, et al. Metabolic and hemodynamic evaluation of gliomas using positron emission tomography. J Nucl Med 1987;28:1123-33 Medline

24. Schramm P, Xyda A, Klotz E, et al. Dynamic CT perfusion imaging of intra-axial brain tumours: differentiation of high-grade gliomas from primary CNS lymphomas. Eur Radiol 2010;20:2482-90 CrossRef Medline

25. Noguchi T, Yoshiura T, Hiwatashi A, et al. Perfusion imaging of brain tumors using arterial spin-labeling: correlation with histopathologic vascular density. AJNR Am J Neuroradiol 2008;29:688-93 CrossRef Medline

26. Tourdias T, Rodrigo S, Oppenheim C, et al. Pulsed arterial spin labeling applications in brain tumors: practical review. J Neuroradiol 2008;35:79-89 CrossRef Medline

27. Cha S, Yang L, Johnson G, et al. Comparison of microvascular permeability measurements, $\mathrm{K}$ (trans), determined with conventional steady-state T1-weighted and first-pass T2*-weighted MR imaging methods in gliomas and meningiomas. AJNR Am J Neuroradiol 2006;27:409-17 Medline

28. Kelly PJ. Computed tomography and histologic limits in glial neoplasms: tumor types and selection for volumetric resection. Surg Neurol 1993;39:458-65 CrossRef Medline

29. Earnest F 4th, Kelly PJ, Scheithauer BW, et al. Cerebral astrocytomas: histopathologic correlation of MR and CT contrast enhancement with stereotactic biopsy. Radiology 1988;166:823-27 CrossRef Medline

30. Greene GM, Hitchon PW, Schelper RL, et al. Diagnostic yield in CT-guided stereotactic biopsy of gliomas. J Neurosurg 1989;71: 494-97 CrossRef Medline

31. Nagashima G, Suzuki R, Hokaku H, et al. Graphic analysis of microscopic tumor cell infiltration, proliferative potential, and vascular endothelial growth factor expression in an autopsy brain with glioblastoma. Surg Neurol 1999;51:292-99 CrossRef Medline

32. Pronin IN, Holodny AI, Petraikin AV. MRI of high-grade glial tumors: correlation between the degree of contrast enhancement and the volume of surrounding edema. Neuroradiology 1997;39: 348-50 CrossRef Medline

33. Pronin IN, McManus KA, Holodny AI, et al. Quantification of dispersion of Gd-DTPA from the initial area of enhancement into the peritumoral zone of edema in brain tumors. J Neurooncol 2009;94: 399-408 CrossRef Medline

34. Iranmahboob A, Peck KK, Brennan NP, et al. Vascular reactivity maps in patients with gliomas using breath-holding BOLD fMRI. J Neuroimaging 2016;26:232-39 CrossRef Medline
35. Agarwal S, Sair HI, Pillai JJ. The resting-state functional magnetic resonance imaging regional homogeneity metrics, Kendall's coefficient of concordance, regional homogeneity and coherence, regional homogeneity are valid indicators of tumor-related neurovascular uncoupling. Brain Connect 2017;7:228-35 CrossRef Medline

36. Agarwal S, Lu H, Pillai JJ. Value of frequency domain resting-state functional magnetic resonance imaging metrics amplitude of lowfrequency fluctuation and fractional amplitude of low-frequency fluctuation in the assessment of brain tumor-induced neurovascular uncoupling. Brain Connect 2017;7:382-89 CrossRef Medline

37. Choi SH, Kim JW, Chang JS, et al. Impact of including peritumoral edema in radiotherapy target volume on patterns of failure in glioblastoma following temozolomide-based chemoradiotherapy. Sci Rep 2017;7:42148 CrossRef Medline

38. Bartolomei F, Bosma I, Klein M, et al. How do brain tumors alter functional connectivity? A magnetoencephalography study. Ann Neurol 2006;59:128-38 CrossRef Medline

39. Maesawa S, Bagarinao E, Fujii M, et al. Evaluation of resting state networks in patients with gliomas: connectivity changes in the unaffected side and its relation to cognitive function. PLoS One 2015; 10:e0118072 CrossRef Medline

40. Esposito R, Mattei PA, Briganti C, et al. Modifications of defaultmode network connectivity in patients with cerebral glioma. PLoS One 2012;7:e40231 CrossRef Medline

41. Harris RJ, Bookheimer SY, Cloughesy TF, et al. Altered functional connectivity of the default mode network in diffuse gliomas measured with pseudo-resting state fMRI. J Neurooncol 2014;116: 373-79 CrossRef Medline

42. Park JE, Kim HS, Kim SJ, et al. Alteration of long-distance functional connectivity and network topology in patients with supratentorial gliomas. Neuroradiology 2016;58:311-20 CrossRef Medline

43. Hillman EM. Coupling mechanism and significance of the BOLD signal: a status report. Annu Rev Neurosci 2014;37:161-81 CrossRef Medline

44. Araque A, Parpura V, Sanzgiri RP, et al. Tripartite synapses: glia, the unacknowledged partner. Trends Neurosci 1999;22:208-15 CrossRef Medline

45. Hervey-Jumper SL, Berger MS. Maximizing safe resection of lowand high-grade glioma. J Neurooncol 2016;130:269-82 CrossRef Medline

46. Duffau H. Long-term outcomes after supratotal resection of diffuse low-grade gliomas: a consecutive series with 11-year follow-up. Acta Neurochir (Wien) 2016;158:51-58 CrossRef Medline 